\title{
Un arrêt du Tribunal fédéral suscite de nombreux échos dans le BMS
}

Bruno Kesseli

L'arrêt du Tribunal fédéral du 3 novembre 2006 concernant l'assistance au suicide a suscité de multiples réactions aussi bien du public que de la presse spécialisée, réactions qui permettent en quelque sorte de mesurer l'importance de ce thème sur le plan politique et dans la société. L'importance de la thématique «suicide - assistance au suicide», notamment dans le cas des personnes atteintes de troubles psychiques, est telle pour le corps médical qu'elle a trouvé un large écho dans le Bulletin des médecins suisses. Cinq articles sont déjà parus ces derniers mois dans le BMS à la suite de l'arrêt du Tribunal fédéral susmentionné, un sixième est publié dans la présente édition et un autre le sera dans une prochaine édition. Dans ce contexte, le bref aperçu qui suit montre que les auteurs ont traité ce thème sous des aspects très divers.

Dans leur article [1], René Raggenbass et Hanspeter Kuhn se sont tout d'abord penchés sur la question de savoir si le Tribunal fédéral avait, dans son arrêt, effectivement introduit le droit à l'assistance médicale au suicide, ainsi que l'avait suggéré une partie de la presse spécialisée et grand public (un article partageant cet avis a également paru dans le BMS [2]). Raggenbass/ Kuhn ont clairement répondu à cette question par la négative sur la base des considérants du Tribunal fédéral, en précisant sans équivoque $\mathrm{qu}^{\prime}$ «aucun médecin n'est tenu de pratiquer l'assistance au suicide, mais [que] l'assistance médicale au suicide n'est plus exclue». Les auteurs justifient en outre la position du Comité central de la FMH au sujet de l'assistance au suicide chez les personnes atteintes de troubles psychiques. Contrairement au Tribunal fédéral qui estime que l'aide médicale au suicide est admissible à certaines conditions soigneusement examinées dans le cas de personnes atteintes de troubles psychiques, le Comité central de la FMH rejette cette idée au motif que les connaissances scientifiques sont encore insuffisantes en ce qui concerne les facteurs déterminants pour une telle approbation.

Dans le commentaire qu'il a rédigé en qualité de juriste [3], Christian Schwarzenegger a souligné que le «droit de mourir» ne peut pas être exigé de l'Etat, donc que l'Etat n'est pas tenu «d'assister activement toute personne à un suicide exempt de douleur au moyen de natrium-pentobarbital». La possibilité de fournir une assistance au suicide aussi chez des personnes souffrant de troubles psychiques, non exclue par le Tribunal fédéral, nécessite à son avis une adaptation des règles de la déontologie médicale. L'argument de Ch. Schwarzenegger est le suivant: comme, en pratique, seul le natrium-pentobarbital entre en considération pour ce type d'aide, les médecins auraient un rôle central à jouer dans le processus décisionnel. «Pour les cas d'assistance au suicide de personnes qui ne sont pas en fin de vie, il n'existe aucune règle déontologique issue des sciences médicales.» Ch. Schwarzenegger estime qu'il faut combler cette lacune en complétant les directives de l'Académie suisse des sciences médicales (ASSM), sinon on devra mettre au point des critères de diligence pour ce genre de cas uniquement par le biais de la jurisprudence.

Dans leur réponse au nom de l'ASSM [4], Claude Regamey et Michelle Salathé ont retenu que l'accompagnement de patients en fin de vie faisait partie des tâches centrales des médecins, mais pas l'offre d'aide au suicide. Au contraire, les médecins sont tenus d'alléger les souffrances qui pourraient sous-tendre le désir de se suicider. La décision d'offrir une telle assistance ne figure pas dans le cahier des charges du médecin mais relève de sa responsabilité personnelle. Ceci dit et vu que le Tribunal fédéral renvoie déjà au devoir de diligence au sens des directives de l'ASSM dans le contexte de la prescription de natriumpentobarbital, l'ASSM renonce sciemment à compléter ses directives éthiques au sens de la demande de Christian Schwarzenegger.

Dans son article [5], Christoph Rehmann-Sutter a partagé l'évaluation des auteurs ci-avant, selon laquelle le Tribunal fédéral n'avait aucunement introduit dans son arrêt un droit à l'assistance médicale au suicide. Annonçant la question essentielle dès le titre, il remet en question la formule $\mathrm{du}$ «droit à sa propre mort» utilisée plusieurs fois par le Tribunal fédéral. Pour Ch. RehmannSutter, il ressort clairement du contexte de l'arrêt en question qu'il s'agit d'une version abrégée du 
droit de chacun, défini de manière plus précise à un autre endroit dudit arrêt, de «décider de quelle manière et à quel moment doit s'achever sa propre vie, pour autant que la personne concernée soit en mesure d'exprimer librement sa volonté et d'agir en conséquence». L'auteur montre que la formule succincte «droit à sa propre mort» pose des problèmes sur le plan de l'éthique juridique, notamment en ce qui concerne l'interprétation de ce droit comme étant un droit au suicide. En conséquence, la Commission nationale d'éthique présidée par Ch. Rehmann-Sutter évite toute formulation de ce genre dans ses prises de position et relève que «chaque être humain est libre de se suicider».

Dans la prise de position publiée ci-après, le comité de la Société suisse de psychiatrie forensique salue l'arrêt du Tribunal fédéral. Il estime positif que ledit Tribunal «ait discuté soigneusement et globalement la problématique de l'assistance au suicide pour les personnes atteintes de troubles psychiques». Selon cet organe de spé- cialistes, l'arrêt du Tribunal fédéral permet, conjointement avec les directives de l'ASSM et la recommandation n ${ }^{\circ} 9$ d'avril 2005 de la Commission nationale d'éthique, «de définir un cadre limitant la crainte d'une instrumentalisation, en particulier des psychiatres».

\section{Bibliographie}

1 Raggenbass R, Kuhn HP. L'aide au suicide n'est pas un droit humain. Bull Méd Suisses. 2007;88(11): 455-6.

2 Taverna E. L'option de l'homicide. Bull Méd Suisses. 2007;88(16):726.

3 Schwarzenegger C. Das Mittel zur Suizidbeihilfe und das Recht auf den eigenen Tod. Schweiz Ärztezeitung. 2007;88(19):843-6.

4 Regamey C, Salathé M. Ärztinnen und Ärzte sind nicht Experten für den freiwilligen Tod. Schweiz Ärztezeitung 2007;88(24):1051-2.

5 Rehmann-Sutter C. Was bedeutet das «Recht auf den eigenen Tod»? Schweiz Ärztezeitung. 2007; 88(25):1109-12. au suicide d'une personne atteinte de troubles psychiques (prescription obligatoire de natrium-pentobarbital)

\section{Martin Kiesewetter, Zurich}

Pour le Comité de la Société Suisse de Psychiatrie Forensique SSPF

* Position non prise dans l'unanimité.

Correspondance: Dr Josef Sachs

Président de la Société Suisse de Psychiatrie Forensique

Psychiatrische Dienste Aargau AG Klinik Königsfelden

Case postale 432

CH-5201 Brugg
Dans son jugement 2A.48/2006 du 3 novembre 2006, la II ${ }^{\mathrm{e}}$ Cour de droit public du Tribunal fédéral s'est penchée sur la possibilité de supprimer l'obligation de prescription médicale pour le natrium-pentobarbital en cas de demande de suicide assisté émanant d'une personne atteinte de troubles psychiques. En se référant aux principes fondamentaux des lois fédérales, le Tribunal fédéral a confirmé le refus clair, sur ce point, des autorités cantonales compétentes.

Dans ses considérants, le Tribunal fédéral s'est longuement penché sur la problématique de l'assistance au suicide des personnes atteintes de troubles psychiques. Il a établi que la personne désireuse de mourir ne pouvait se prévaloir du droit au suicide assisté. Il a en outre constaté que l'Etat avait un devoir fondamental de protection du droit à la vie, mais que ce devoir n'allait pas, en règle générale, jusqu'à s'opposer à la volonté expresse de l'intéressé capable de discernement.

Si le Tribunal fédéral reconnaît parfaitement que le droit à l'autodétermination au sens de l'art. 8 al. 1 CEDH inclut le droit de décider de la manière et du moment de mettre fin à sa vie (dans la mesure où l'intéressé est capable de se forger sa propre volonté et d'agir en conséquence), c'est cependant le devoir de l'Etat de «s'assurer, par une procédure adéquate, qu'une éventuelle décision de mettre fin à ses jours traduit bien la libre volonté de l'intéressé». Mais il s'agit aussi de lier la licéité de l'aide au suicide à la «connaissance de l'état de santé de l'intéressé» et de soumettre la remise d'une substance dangereuse en vue du suicide aux conditions de sa 
prescription dans le cadre des «règles admises des sciences médicale et pharmaceutique et de la connaissance de l'état de santé de l'intéressé».

Même si une assistance au suicide, ou la prescription de natrium-pentobarbital, à des personnes atteintes de troubles psychiques incurables, durables et graves n'est "plus obligatoirement contre-indiquée ni considérée dans tous les cas comme une violation du devoir de diligence médical», une "prudence extrême est de mise: il s'agit de faire la distinction entre le désir de mourir, qui est l'expression d'un trouble psychique qu'il est possible et nécessaire de traiter, et la décision librement choisie, mûrement réfléchie et durablement décidée d'une personne capable de discernement [...], qu'il convient, le cas échéant, de respecter. Si le désir de mourir repose sur une décision autonome, prenant en compte l'ensemble de la situation, il est éventuellement permis d'apporter une assistance au suicide à des personnes aussi atteintes de troubles psychiques $[\ldots] »$.

De l'avis du Tribunal fédéral, une telle appréciation suppose «nécessairement la présence d'une expertise psychiatrique approfondie». Cette expertise doit reposer sur un examen psychiatrique minutieux et un diagnostic soigneux et exige «un long accompagnement médical par un spécialiste, disposé, le cas échéant, à établir une ordonnance médicale basée sur cette observation».

Après le jugement du Tribunal fédéral, les milieux médicaux ont exprimé la crainte que l'on puisse faire pression sur les psychiatres, en particulier par l'entremise des organisations d'aide au décès, pour qu'ils acceptent d'effectuer ces expertises, voire qu'il se trouve même des experts pour se laisser guider «plutôt par le désir de mourir d'un patient» que par la volonté «d'évaluer à fond sa décision» - pour citer le Tribunal fédéral «selon des critères scientifiques, quant à sa légitimité médicale».

La crainte a aussi été exprimée qu'une pression puisse s'exercer sur les médecins pour qu'ils considèrent le désir de mourir d'une personne atteinte de troubles psychiques, de manière générale, comme l'expression du droit de l'intéressé à l'autodétermination et avant tout sous l'angle de la faculté momentanée de discernement, mais sans accorder l'importance nécessaire à des critères tels que l'obligation d'une décision prise après mûre réflexion et durable. Dans ce contexte, il est loin d'être exclu que les psychiatres soient instrumentalisés.

Dans la continuité de ces préoccupations, le Comité de la Société Suisse de Psychiatrie Forensique a pris la position suivante: Il salue le jugement du Tribunal fédéral du 3 novembre 2006, qui a le mérite de discuter soigneusement et globalement la problématique de l'assistance au suicide pour les personnes atteintes de troubles psychiques.

Il apprécie en particulier qu'il soit expressément mentionné, dans le jugement, que

- dans le «difficile conflit» entre l'assistance au suicide, qui ne fait pas partie de l'activité médicale, et le «respect de la volonté du patient», il faut aussi respecter la décision du médecin prise en conscience «dans des situations limites»;

- l'aide au suicide, comprise comme devoir librement consenti par le médecin, ne peut pas lui être imposée;

- le médecin qui décide de prêter assistance au suicide porte aussi la responsabilité d'avoir respecté en tout point son devoir de diligence médical et de ne pas s'être laissé guider principalement par le désir de mourir de son patient;

- une expertise psychiatrique indépendante portant sur la question de la volonté libre, mûrement réfléchie et durable, manifestée sans pression extérieure, de la personne désireuse de mourir, doit être impérativement diligentée;

- la responsabilité d'administrer du natriumpentobarbital ne peut être déléguée à une organisation d'aide au décès.

Dans ce contexte, le comité de la Société Suisse de Psychiatrie Forensique constate ce qui suit: Celui qui est prêt à assister une personne atteinte de troubles psychiques dans son désir de suicide ne peut se fonder sur la seule faculté de discernement momentanée du candidat à la mort.

Il lui faut aussi apporter la preuve que le désir de mourir est indépendant de l'influence de tiers, et répond à une décision mûrement réfléchie et durable. La prise de position soigneuse et conforme aux règles de l'art n'obéit pas seulement au devoir fondamental du médecin d'éviter tout dommage à son patient, mais constitue aussi la condition nécessaire pour protéger le médecin prescripteur de natrium-pentobarbital de conséquences pénales et ressortissant de son devoir d'appréciation et de contrôle (à ce propos, il faut rappeler que c'est la tâche du médecin prescripteur de vérifier la qualité et la validité de l'expertise).

La prise de position d'un expert sur la question de la faculté de discernement, de la mûre réflexion et de la durabilité d'un désir de suicide telle que l'exige le Tribunal fédéral ne suppose pas seulement une longue période d'observation, mais aussi une compétence professionnelle 
en la matière. Pour établir une telle expertise, il importe aussi de se pencher longuement sur ce que laissent entrevoir le pronostic de la pathologie et l'évolution du patient ainsi que les possibilités thérapeutiques (même si elles n'ont pas été prise en compte jusqu'à présent mais peuvent être raisonnablement envisagées). Il s'agit aussi de discuter à fond et avec soin les facteurs sociaux et d'examiner ce qui a été fait et tenté dans le passé en matière thérapeutique et quels fardeaux d'éventuelles nouvelles procédures thérapeutiques imposeraient à l'intéressé.

C'est précisément parce qu'une expertise sur la faculté de discernement, la décision mûrement réfléchie et durable implique de prendre en considération la position du médecin ou du psychologue traitant sur la situation thérapeutique, qu'une expertise, mandatée par une organisation d'assistance au suicide et qui ne prendrait pas en compte ces aspects ainsi que l'éclairage apporté par la science psychiatrique, devrait être considérée comme insuffisante.

Une obligation médicale de prescrire du natrium-pentobarbital n'existe pas, même si on reconnaît un droit fondamental à l'autodétermination à une personne exprimant une volonté de mourir mûrement réfléchie, durable et non symptomatique de troubles psychiques.

La prescription médicale de natrium-pentobarbital apparaît hautement problématique à la lumière de la déontologie médicale si - indépendamment de l'opinion subjective, mais capable de discernement de la personne décidée à mourir (qu'elle soit juste ou non) - il existe des moyens thérapeutiques d'améliorer sa situation. En tout cas, elle aussi implique une discussion et une justification minutieuses. Il n'est pas permis de se laisser guider principalement par le désir de mourir d'un patient sans en vérifier à fond la justification médicale.
Une attention soigneuse portée aux considérations exprimées dans le jugement du Tribunal fédéral du 3 novembre 2006, de même que le respect des directives médico-éthiques de l'Académie Suisse des Sciences Médicales, et de la Recommandation $\mathrm{n}^{\circ} 6$ du 27 avril 2005 de la Commission nationale d'éthique pour la médecine humaine à propos de l'assistance au suicide imposent une prudence extrême dans la prescription de natrium-pentobarbital et dans les expertises qui la justifient.

D'autre part, l'observation de ces directives et du jugement du Tribunal fédéral permet de définir un cadre limitant la crainte d'une instrumentalisation, en particulier des psychiatres.

Celui à qui sa conscience ou la compréhension de son rôle interdisent d'effectuer une expertise sur la faculté de jugement, la mûre réflexion et la durabilité d'un désir de suicide et sur le pronostic et les possibilités évolutives et thérapeutiques pourra se prévaloir de raisons recevables à une telle attitude. Il en va de même pour celui qui y est disposé et qui, dans le respect des règles de l'art, cherche le dialogue avec les spécialistes ayant traité le patient jusqu'à maintenant (la proximité et le dialogue que l'expert recherchera non seulement avec la personne décidée à mourir, mais aussi avec le thérapeute s'opposent à une collaboration «institutionnalisée» avec des organisations d'aide au décès et protègent des manipulations).

Au contraire, celui qui établit ce type d'expertises sans respecter l'état de la jurisprudence et celui de la science psychiatrique (notamment dans le domaine forensique) pourra nuire au patient tout d'abord, à sa spécialité éventuellement et - s'il est appelé à rendre des comptes - à luimême, le cas échéant. 